\title{
Basic clinicians: on the marriage between clinicians and basic scientists at AHRI/ALERT
}

\section{S BRITTON}

Armauer Hansen Research Institute, P.O. Box 1005, Addis Ababa, Ethiopia

In "The Two Cultures", C.P. Snow speculated that the arts and sciences or if you wish, the humanistic versus the scientific approach could never meet. Does this apply also to clinical approaches versus the strictly scientific ones in the biomedical world? The answer is no. The ALERT/AHRI setup is a living example to the contrary. Unlike other speakers at this symposium I shall not base my reasoning to this point on historical aspects but rather focus on the present and even dare to look into the future.

The value of basic science to clinicians

What benefit do clinicians derive from involvement in basic research like that at AHRI? None. The only but paramount point is that they are involved in research activities at all. Such activity, when appropriately performed, will make the clinician a better doctor for his/her patients. It will provide him with a critical mind, it will help him to get oriented in the vast medical literature, to distinguish the false from the real, to arm him against the propaganda - often draped in a seducing scientific outfit - that the drug and medico-technical industry is constantly bombarding him with. Don't get me wrong. It does not make him a good doctor, but it is a crucial component of a good doctor. Thus, in my view it is not the achievements of his research activities that are important but the mere fact that he is involved in any research activity at all. This of course need not be basic research. It may involve merely quality control measurements of regular clinical procedures or simply compiling and evaluating information from published work. Here it is pertinent to mention the vastly underrated impact of pure clinical research based on patient records and collection of clinical data. Such research is time consuming and is constantly exposed to numerous difficulties never faced by 
experimental and basic scientists. It results in few publications and rarely renders the executor famous overnight. We may only remember the non-existance of Nobel prizes awarded for pure clinical research achievements. This unjust balancing of clinical research versus the more basic one must be stopped when it comes to ranking applicants for academic clinical positions, or else we will soon not have any more of it.

\section{The value of clinicians for basic scientists}

What, then, is the value which basic biomedical scientists derive from working with clinicians? None really, in many instances. Their work should consist of disclosing biological processes underlying health and disease and for this they are not directly dependent on clinicians. Most of the important biomedical achievements in the basic sciences have been done in isolation from clinical influences. Monoclonal antibodies and gene cloning are recent examples of this. But clinicians and the biomedical industry, with awareness of the impact of these discoveries, have quickly explored them for clinical purposes.

There is, however, a tendency nowadays to bring together scientists of different disciplines to focus on one defined biomedical problem following the example of the space flight. Rather than having anatomists working in one place and physiologists in another, they are combined with clinicians in one entity to resolve a problem like diabetes. This approach is also introduced in medical education, which is slowly becoming organ-oriented. Students are taught everything about one organ or organ system from basic function to intricate disease symptoms rather than splitting man up into his anatomical, histological and biochemical parts, which is a way he never presents in real medical life.

Thus, there is an as yet unproven hypothesis that by bringing basic scientists of different extractions together with clinicians on a given problem we shall see more rapid and relevant results. The AHRI/ALERT set-up is a very early example indeed of this approach, which is just now beginning to develop on a large scale in the so called developed world. Has it been a meaningful construction, i.e. has it provided results in the interests of the patients that would not 
have been obtained otherwise, and has it had mutually beneficial effects on clinicians and basic scientists working in this setting? I am inclined to answer a quiet and modest "yes" to this question.

\section{The AHRI/ALERT construction}

The spectrum of biomedical activities concerning mainly leprosy within the AHRI/ALERT set-up is broad. It comprises the following: epidemiological work done by the Leprosy Control Unit; medical and surgical interventions in the clinical wards, with adjacent service activities like x-ray, clinical chemistry and bacteriology; a vast outpatient service for dermatological disorders, in general, and leprosy and cutaneous leishmaniasis, in particular (ALERT); a clinical pathology service for tissue specimens, and a basic research unit (AHRI), equipped to do experimental laboratory research, utilizing the latest molecular and cellular immunology techniques, including experiments on laboratory bred animals. What, then, has emanated from this, at the time, futuristic contruction?

In the early seventies the lymphocyte transformation test (LTT) was established at AHRI, and it has been a valuable adjunct to the clinical scoring of leprosy patients in particular when they are moving along the scale. The treatment of reversal reaction (RR) with high doses of steroids in fact derives its rationale from experiments utilizing lymphocytes from RR patients in the LTT assay. The recent but still disputed finding from AHRI scientists that the lymphocyte growth hormone IL-2 may indeed restore the responsiveness of lepromatous leprosy patients to $M$. leprae opens up a new avenue for treating these patients. Thus, there are achievements that have been of direct benefit in the diagnosis and treatment of leprosy patients. But, admittedly, it is meagre considering the amount of money and personnel, the hectolitres of spilled blood and killed mice that it has required. We really have to request more of it before we can call this set-up a success that can be transferred to other health sectors. 
Prospects for increased contacts between clinicians and basic

\section{scientists}

In order for an arrangement like AHRI/ALERT to function optimally, there has to be intimate and daily contact between epidemiologists, clinicians and basic scientists, and this has certainly not been the case up to now. The fault lies mainly with the basic scientists. Their language is unnecessarily complex, often used to hide fairly trivial information, but still enough to keep a somewhat shy and tired clinician away. Indeed we also see this phenomenon of the Emperor's new clothes among us basic scientists. The new armada of molecular biologists and geneticists that has now luckily - also invaded AHRI speaks a pompous scientific language that we conventional and seemingly outdated cellular immunologists do not dare to disrobe. Thus, there is room for a simplified scientific language that can be understood by all disciplines and through our regular joint (AHRI/ALERT) seminar activities, I believe we are beginning to get there.

I also think that we must be careful to balance researchers with clinical experience to those with a more purely natural science background. At present there is fifty percent of each. This seems to be a working proportion. It would also be important in the recruitment of medical personnel to ALERT to choose those with expressed and possibly documented scientific interest.

In order to create contacts there have to be meeting points and there has to be a mutual feeling of mutual benefit from such contacts. When I say benefit, I mean it in the crude sense as well, i.e. that the clinicians can smell publications and the basic scientists' material and information that may lead to the compilation such publications.

\section{Epidemiology}

Clearly the weakest point of cooperation at AHRI/ALERT has been the epidemiological aspect of leprosy which has mainly been pursued so far by the Leprosy Control Unit. This can be explained on AHRI's part by the lack of personnel with epidemiological experience or interest. Further, the Leprosy Control people are mostly out in the field and thus cannot take part in whatever intellectual conversation is going on 
at the AHRI/ALERT campus. This is clearly a disadvantage for both parties.

For example, the MDT programme for the multidrug treatment of leprosy has been introduced on a worldwide scale through the initiative of wHO without the slightest insurance of a scientific evaluation of this major endeavour. One of the best stratified areas for the MDT programme is the shoa region covered by the Leprosy Control Unit at ALERT. However, the option to have a controlled trial between monotherapy and MDT is rapidly fading with the overall introduction of the MDT approach. Also, we have no elaborate plans (on the AHRI's side) for investigating patients released from MDT treatment but relapsing. Are they genetically distinct from the non-relapsing population or do they harbour bacilli less sensitive to the drugs or less prone to activate a protective immune process?

We believe that our initial successes at AHRI in detecting, with methods applicable in the field, an antigen in the urine of patients with a high bacillary load will offer such a mutually beneficial tool that will boost the contacts between the epidemiologists and the basic scientists. There is also a need for a less unidirectional recruitment policy at AHRI, which would allow the hiring of epidemiologists in parallel with more conventional basic scientists.

\section{Histopathology}

A fruitful meeting point over the last year, for clinicians and surgeons as well as internists and basic scientists has been over the last year the clinical histopatology unit at AHRI. There, clinicians have been able to look at samples together with the pathologist and jointly discuss the morphological picture in relation to the clinical findings. I believe this has been the most important single contribution to the markedly increased contacts and interactions between clincians and basic scientists that have taken place at AHRI/ALERT. Although the histopathologist is in many ways clinically oriented, his links with the basic sciences are strong and he can introduce the clinicians at AHRI to them. The accessibility to the clinicians of the pathologist in a scientific setting is certainly worth recommending as it is 
a transition area where the clinicians are at ease and can use as

a starting point on their way to the experimental sciences.

\section{Parasitology}

Another area where clinicians are beginning to show an increased interest is in the culturing of leishmania promastigotes from lesions that are difficult to classify clinically or histopathologically. To see the actual pathogen apparently not only helps the diagnosis but also increases the interest on the part of the clinicians for the immunopathology of the disease and for the option of drug testing on the parasites. We believe the same will be true in leprosy when we can grow individual bacterial isolates in nude mice and then test these isolates functionally in various in vitro assays. The correlation between clinical appearance and individual bacterial characteristics is something that should attract the interest of clinicians.

\section{Molecular immunology}

Over the last year molecular biology techniques have been introduced at AHRI and so far the clinicians have been rather cool in their attitude to these approaches to the diagnosis, characterization and control of leprosy and leishmaniasis. But this state of affairs can not continue, because molecular biological approaches require a cooperation between the molecular biologist and the cellular immunologist to test whatever products that are obtained by the western blot or gene cloning techniques. In this cooperative atmosphere it is much easier to take the next step of going to the clinician to discuss the prospect of testing the material in vivo. At AHRI too, we have taken a short cut by recruiting two Ethiopian M.D.'s with previous experience as health officers, for post-doctoral training. These have been immediately introduced into the world of the molecular biology of leprosy and leishmaniasis, and we hope that they will ensure the necessary links with clinical medicine.

\section{Sense of moral commitment}

Thus, even if there are no proven major effects to the benefit of patients with leprosy or any other disease, of cooperation between clinicians and basic scientists, we certainly hold the 
view that they exist. In addition, the many new channels of communication that have been opened over the last year have certainly created a more relaxed but yet intellectual atmosphere on the campus.

When involving more and more people in the basic sciences though, we must never forget that what we are doing is biological history. All the small bits and pieces that we are disclosing have already been in operation for millions of years. We do not make any new discoveries. We just explore ground that has already been traversed. 